\title{
Translational systems biology understanding the limits of animal models as predictors of human biology
}

\author{
Carine Poussin', Leonidas Alexopoulos ${ }^{2}$, Vincenzo Belcastro', Erhan Bilal ${ }^{3}$, Carole Mathis', Pablo \\ Meyer $^{3}$, Raquel Norel ${ }^{3}$, Jeremy J Rice ${ }^{3}$, Gustavo Stolovitzky ${ }^{3}$, Julia Hoeng', Manuel Peitsch ${ }^{1 凶}$ \\ 'Philip Morris Research and Development, Neuchatel, Switzerland \\ 2Protatonce Ltd, Glyfada, Greece \\ ${ }^{3}$ BMM, New York, United States
}

\section{Motivation and objectives}

Inferring how humans respond to external cues such as drugs, chemicals, viruses or hormones is an essential question in biomedicine. Very often, however, this question cannot be addressed since it is not possible to perform experiments in humans. A reasonable alternative consists of generating responses in animal models and "translating" the results to humans. The limitations of such translation, however, are far from clear, and systematic assessments of its actual potential are urgently needed.

A series of challenges have been designed in the context of the 'sbv IMPROVER' project (Industrial Methodology for Process Verification in Research; http://sbvimprover.com/) to address the issue of translatability between humans and rodents. Our aim is to understand the limits and opportunities of species to species translatability at different levels of biological organization: signalling, transcriptional, and release of secreted factors (such as cytokines, chemokines or growth factors).

\section{Methods}

Normal Bronchial Epithelial Cells of both human and rat origin were selected to address this question. The cells were exposed to more than 50 different substances and for each stimulus, samples were collected at different time points to generate phosphoproteomic (After 5 and $25 \mathrm{~min}$ stimulus exposure), gene expression (after 6 hours) and secreted protein (after 24 hours) data.

Our challenge will provide participants with both training and test data sets which are designed to assess the ability of methods to predict the responses in Normal Human Bronchial Epithelial cells, from the responses observed in Normal Rat Bronchial Epithelial primary cells.
The central questions that we will pose in this challenge are: (1) Can the phosphoproteomic responses induced by stimuli addressing several distinct signalling pathways in human cells be predicted given the responses generated with the same stimuli in rat cells? How does the accuracy of the prediction depend on the nature of the applied perturbation? (2) Which gene expression regulatory processes (biological pathways / functions) are translatable and therefore predictable across species, and which are too divergent?

\section{Results and discussion}

We will present the community with questions and large scale omics data aimed at assessing methodologies designed to infer human biology from non-human biology.

The sbv IMPROVER project, the website, and the Symposium are part of a collaborative project designed to enable scientists to learn about and contribute to the development of a new crowd sourcing method for verification of scientific data and results.

\section{Acknowledgements}

The project team includes scientists from Philip Morris International's Research and Development department and IBM's Thomas J. Watson Research Center. The project is funded by PMI.

\section{References}

Meyer P, Hoeng J, Rice JJ, Norel R, Sprengel J et al. (2012). Industrial methodology for process verification in research (IMPROVER): toward systems biology verification. Bioinformatics 28(9), 1193-201. doi:10.1093/bioinformatics/. btș!nto

Meyer P, Alexopoulos LG, Bonk T, Califano A, Cho CR, et al. Verification of systems biology research in the age of collaborative competition (2011). Nat Biotechnol 29(9):811-5. doi:10.1038/nbt.1968 